\title{
Requirements Identification for the Development of a Design Involving Adaptive ICTs for Supporting Bipolar Disorder Treatment in Different Healthcare Contexts
}

\author{
Emanuele Torri \\ APSS, Trento, Italy \\ emanuele.torri@apss.tn.it
}

\author{
Oscar Mayora \\ CREATE-NET, Italy \\ omayora@create-net.org
}

\author{
Jesus Cobo, Diego Palao \\ CSPT, Spain \\ (jcobo,dpalao)@tauli.cat
}

\begin{abstract}
This paper presents the patient and caregiver perspectives on ICTs (information and communication technologies) supporting bipolar disorder management in multinational healthcare provisioning contexts. The envisioned mHealth (mobile health) solutions should incorporate general requirements that can be instantiated into different clinical settings. The involvement of users in designing new technologies for mental health is crucial in ensuring patient empowerment and the patient-centeredness of services. We conducted focus groups to understand user needs, attitudes, and experiences with regard to the supportive ICTs in the two target regions where the expected solutions will operate. The survey results provided valuable inputs for the formulation of the clinical requirements for the development of a transnational call for tender concerning mHealth solutions aimed at supporting treatment for bipolar disorder. This tender was open to public purchasers in different European countries. The study was part of the NYMHPA-MD (Next Generation Mobile Platform for Health in Mental Disorders) project, which was co-funded by the European Commission.
\end{abstract}

Keywords: Management, Design, Human Factors, Bipolar Disorder, Healthcare contexts, mHealth

\section{INTRODUCTION}

Bipolar disorder is a common mental disorder. According to available epidemiological evidence, the prevalence of this complex mood disorder in the population is $1 \%-2 \%$. The disease is associated with considerable clinical, social, and economic hardships [1].

People with bipolar disorder experience pathological mood episodes that fall into two broad categories: mood episodes characterized by symptoms of mania (euphoria) and mood episodes characterized by symptoms of depression. The disorder has a typical relapsing remitting course and is associated with a high risk of relapse and hospitalization. Bipolar disorder is often a long-term and persistent illness, whose management can span many years. Many patients do not regain the level of psychological functioning they had before they developed the disorder.

The risk of experiencing a new episode in bipolar disorder can be reduced significantly by treatment with lithium or other mood stabilizers. Medical treatment may be enhanced by psychoeducation, cognitive behavioural therapy, and peer support practices [2] [3] [4] [5]. Early detection of prodromal depressive and manic episodes and adherence to treatments are essential for preventing the progressive development of the disease [2]. Treatment needs to be personalized and tailored to the specific individual and should be based on the patient as a whole.

Quality of care depends on the quality of the available information, the patient's level of self-management, and the systematic engagement between the patient and informal caregivers. Users' involvement in mental health has been described as a practice that is necessary ethics-based, and evidence-based [6]. It encapsulates a range of different ideas (as does the concept of "user" itself), right from active participation of the individual in decision-making at the micro level, to a more macro-level involvement in service planning and evaluation and, increasingly, in the areas of training and research [7].

In the case of bipolar disorder, there is an emerging shift in the illness paradigm, namely, a shift from a focus on mood episodes to a focus on inter-episodic mood instability.

According to the World Health Organization, the use of mobile and wireless technologies to support the achievement of health objectives - mHealth (mobile health) - has the potential to transform health service delivery and the quality of care across the globe. Through sensors and mobile applications, mHealth allows the collection of considerable medical, physiological, lifestyle, social interaction, and daily activity data. This can serve as a basis for evidence-driven care practices while facilitating patients' access to their health information anywhere and at any time. Further, mHealth can support clinicians in treating patients more efficiently as mobile applications can encourage adherence to a healthy lifestyle, to certain behaviours, and to taking the necessary medications, resulting in more personalized care.

Studies on mobile phone applications in mental health have shown promising results in terms of reducing depressive symptoms, stress, anxiety, and substance use [8]. In recent years, the growth in e-mental health technologies has increased, and the number of electronic platforms for self-monitoring in the area of mental health, including bipolar disorder, is rapidly increasing [9]. Recently, the electronic self-monitoring of the affective symptoms of bipolar patients, which involved the use of cell phones to prompt patients to respond to weekly text messages, was proposed as an easy and inexpensive way to monitor and identify early signs of emerging affective episodes so that providers could intervene shortly after the prodromal symptoms appeared [2]. Among these e-mental health technologies, in the MONARCA (MONitoring, treAtment, and pRediCtion of bipolAr Disorder Episodes) project, solutions were developed for the 
multiparametric long-term monitoring of behavioral and physiological information (i.e., mood/irritability, sleep, speech, and physical activity) relevant to bipolar disorder and for treatment adherence in a bidirectional feedback loop between patients and providers [2].

While the opportunities for treatment enabled by mHealth seem promising, the integration of such tools in different psychiatric contexts of care is not straightforward [10]. The adaptation of platforms to satisfy all requirements (including clinical and organizational in addition to issues related to ethics, data, safety, and privacy) in diverse environments and meeting the expectations of different stakeholders (clinicians, technologists, researchers, policymakers, patients, and informal caregivers) is, indeed challenging.

Understanding the user's perspective right from the preliminary stages of the designing of new mobile technologies is crucial for laying a strong foundation for the establishment of the requirements of services that can be instantiated into mental health settings.

This paper presents the preliminary results of a multinational survey conducted within the scope of the NYMPHA-MD (Next Generation Mobile Platform for Health in Mental Disorders) project. The survey aimed to identify common recommendations for the requirements of mHealth applications.

\section{HEALTHCARE CONTEXTS IN THE NYMPHA-MD PROJECT}

The NYMPHA-MD project is a PCP (pre-commercial procurement) initiative, funded by the European Commission under the Seventh Framework Program. The NYMPHA-MD project focuses on the implementation of a PCP plan of mobile ehealth services and targets three European regions, namely, Trentino (Italy), Catalonia (Spain), and Copenhagen (Denmark). A tender for such services would need to satisfy the requirements established by the public purchasers from the three regions. It should also allow for the sustainable implementation of the technological solution aimed at empowering patients and enabling patient-centered care through new mobile eHealth services.

Currently, in each of the three target regions, different approaches with regard to treatment, management, and support for patients with bipolar disorder are being implemented. In mental health, different clinical and scientific perspectives draw on many and complex medical, professional, organizational, cultural, legal, and social factors.

Taulí Mental Health Center in Catalonia provides mental health services to a population of nearly half a million in the Area of Vallès Occidental, which is a primarily residential area in the city of Sabadell (province of Barcelona, Catalonia). These services include a special department for child and adolescent mental health, two outpatient centers for adult mental health, an inpatient unit for adults, a child and adolescent inpatient unit, day hospitals, and other rehabilitation and community services.

In Trentino, the Department of Mental Health of Trento has a catchment area of nearly 150,000 inhabitants, and this is the main residential area in the town of Trento. The mental health center is the hub of the community-based system of care, which operates on the basis of the recovery-based philosophy of "doing together."
The Department of Mental Health manages all adult psychiatryrelated activities/centers for the cure and rehabilitation of mental disorders. These activities/centers include hospital wards, outpatient centers, day centers, home care services, and residential facilities. For years, what was being implemented was a broad peer support initiative, wherein around 45 expert users and family members (in Italian, Utenti e Familiari Esperti [UFE]) were engaged in day-to-day activities with the patients while working alongside medical and nonmedical professionals. The initiative also included in-service provision and improvement.

The mental health services in region $\mathrm{H}$ in Copenhagen are provided through six psychiatric centers for outpatient treatment. The outpatient services are provided through the following means: through general practitioners in the case of patients who have long-term, stable conditions (which require monotherapy); private psychiatrists (who deal with few hospitalization cases and cases with no comorbidity); a community psychiatric center (which deals with people who are unstable, those that have comorbidity, and those that require frequent hospitalization); and a regional clinic for affective disorders (this clinic is for the management of the onset of a single manic episode/bipolar disorder [early intervention], treatment resistance or rapid cycling, and pregnancy).

The innovative mHealth device envisioned in the NYMPHA-MD project would enable continuous clinical monitoring and patient engagement in order to dynamically support illness management and potentially identify early deviations in mood and attitudes suggesting the onset of a crisis, thereby allowing early and personalised management. The mobile application should enable a direct relationship among the psychiatrist, other professionals, and caregivers. We needed to conduct a timely and flexible investigation of business cases to match potential solutions with real expectations and demands of users in different contexts. As part of the methodology to define the requirements of the NYMPHA-MD call for tender, apart from surveying the user's perspective, we conducted interviews and face-to-face meetings with other stakeholders in order to collect information to define technical and clinical standards for mobile applications in the three target regions. The outcomes of this process are beyond the scope of this paper, which focuses only on the user's perspective in Italy and Spain.

\section{METHODS}

We conducted some focus groups, as they provide detailed information concerning respondents' beliefs, attitudes, feelings, and experiences. Focus groups can promote collaboration with researchers and the involvement of participants in the decisionmaking process, enabling the participants to be empowered and valued as experts [11].

In 2014, independent focus groups with patients with bipolar disorder were carried out in the target locations of Trentino and Catalonia. In Copenhagen, numerous workshops and focus groups for gaining an insight into patients' expectations and suggestions were performed before 2014. The results of these workshops and focus groups are not reported here, but are available in the literature [12] [13] [14].

To obtain information about the mental health services in Trento and Sabadell, we conducted unstructured group interviews on the following topics: (a) knowledge and current and future use of 
mHealth technologies in mental health settings; (b) attitudes, expectations, advantages, disadvantages, and hindrances in the use of such technologies for the management of bipolar patients; and (c) objectives, advantages, and challenges of the NYMPHA-MD project from the patient's perspective. To stimulate discussion among the participants, we presented some actual cases. An experienced external moderator, supported by a mental health professional, led the discussion and facilitated interactions among people.

We recruited participants from the purposeful sample of the larger population of patients and caregivers in Trento and Sabadell.

In particular, in Sabadell, we conducted numerous meetings with a group of more than 20 expert patients affected by bipolar disorder and some selected caregivers, who were available to join the group. Most of the patients and caregivers were leaders or active members of the local association of bipolar patients. The members of this group had vast and broad expertise with the illness and its consequences, on account of their personal experience and an intensive educational program that was implemented regularly in the area. The group also included some close family members and caregivers. In Trento, we conducted three meetings, wherein the participants were around 15 patients and caregivers who had a long history of experience with bipolar disorder and its treatment; these patients and caregivers were selected from among members of the psychoeducational groups mentioned earlier. In the focus groups, the participants were five expert users and family members; these expert users and family members were selected from among individuals employed for the management of acute crisis."

In a final joint workshop held in Sabadell to gather data and establish a common knowledge base, a dedicated focus group was conducted. The participants of the group were 10 expert patients from the Trento area (recruited from among expert users and family members) and the Sabadell area (recruited from the local bipolar disorder association). During the focus group, we addressed, shared, grouped, and reviewed the main issues that emerged from the previous independent meetings, with the aim of establishing some overall recommendations.

Each focus group session was audio taped and later transcribed verbatim. The transcribed focus group discussions were then analyzed using qualitative content analysis.

\section{PRELIMINARY RESULTS}

The feedbacks and recommendations for designing a suitable mHealth device for the management of bipolar disorder, obtained from the discussion (on the topics mentioned earlier) during the focus groups can be grouped and summarized as follows.

\section{Continuous monitoring}

- The mHealth device should enable the detection of bipolar episodes in the early phase, when prodromal symptoms of the relapse (both mania and depression) arise.
- It should include: (a) aspects of sleep control and circadian rhythms and should also include features that enable the self-monitoring of hours of sleep and daily activity; further, (b) it should enable the detection of awakenings in the night or the reduction of hours of sleep.

- It should enable the collection of subjective (i.e., mood state) and automatically generated behavioral data (data on mobility, social activity, and physical activity) for the detection of prodromal symptoms of depressive/manic episodes.

- It should include specific items that can be monitored through self-assessment (personalized inputs, medications, etc.); further, patients with special questions or monitoring needs due to special problems (alcohol consumption, binge eating, etc.) should be able to personalize this feature according to their specific needs.

\section{Alert systems and interaction with clinician}

- The mHealth device should have gamification/visualization features with regard to early warning signs and a feature that enables the user to manage 'alarms,' such that he/she is not disturbed.

- It should include interactive text/sound/response systems and should provide the user with some degree of positive reinforcement (diaries, messages, etc.).

- It should include some reminders with alerts related to treatment, doctor's visits, and blood tests (perceived as a very important issue) and should allow the user to establish contact with the mental health system.

- It should allow the user to make 24-hour urgent contact with a professional team in the case of a crisis or decompensation.

- It should include information about medicines (characteristics, secondary effects, safety alerts, etc.), emergency protocols (or should list contact numbers), and resources in the community, with alerts or periodical information from the professional team (physicians, nurses, social workers, etc.).

- It should enable the user to note important events (i.e., life events) for his/her personal records or so that he/she can discuss them later with his/her therapist. 


\section{Social networking}

- The mHealth device should facilitate communication with caregivers (family members and peers) and enable the user to receive coaching from expert users and should also enable group discussions (involving other patients and expert patients).

- It should enable the sharing of static and dynamic resources (i.e., case stories, relevant clinical readings, FAQs, stories, artistic depictions, photos, and videos) with peers and professionals.

- It should enable the user to be in contact (by phone, SMS, or email) with others so that he/she can receive support by peer members and associations and other self-support groups and agencies.

- It should facilitate the peer sharing of information related to social/sports/cultural activities.

- It should allow expert patients to moderate peer groups and engage in the peer sharing of their own experiences so that the users can achieve a better awareness and selfmanage their illness (clinicians and other professionals could moderate other aspects concerning the disorder).

- It should promote a specific approach to "hope": the device should make available to the patient, narratives of "recovery and hope" from expert patients and relatives, which would serve as a strong empowering tool for management programs that can be included in tried and tested ways of coping with the disorder.

\section{Technical features of the mHealth device}

- It should promote access to other patients' narratives containing the message of hope

- It should present data visually and provide a quick overview of monitored data.

- It should be aesthetically pleasing, with a variety of attractive colors, and should also be non-stigmatizing.

- It should be user-friendly (for both patients and clinicians), modern, easy, small, elegant, and attractive.

- It should have high definition and a clear tactile screen, should support various languages, and the text/letters should be adaptable to diverse populations, including older people and people with some degree of visual impairment, who need large text and who need largesized letters.
- It should allow voice service activation in the case of users with mobility impairments.

- It should have a long-lasting battery.

\section{Main concerns}

- Appropriate flow and sharing of information with other people (clinicians or peers) depending on the type of data: For example, information concerning pharmacotherapy treatment should only be shared with the clinician. In other words, it should be ensured that the right information goes to the right person.

- Safety and privacy with regard to the management of the collected information: That is, attention needs to be paid to who will detect the alarm and who will manage the configuration of alert systems/alarms.

- Redundancy of psychoeducational programs: The information provided by the system should complement psychoeducational programs but not substitute them.

- Lack of interpersonal contact/communication: Social networking through electronic devices should not substitute actual networking and should be promoted only as complementary to face-to-face networking. Sharing experiences through actual face-to-face networking facilitates coping with difficult-to-manage situations.

\section{CONCLUDING REMARKS}

In the formulation of requirements for new ICTs, from a patientcentered perspective, listening to what users want and to their opinions is essential for understanding different care contexts, finding recurring themes, and providing support. In developing the clinical framework of the envisioned service solutions, experiential knowledge of patients and informal caregivers can integrate and complement the professional expertise of clinicians, technologists, managers, and others. We believe that technological innovation in mental health should be based on both experiential and professional knowledge.

Service users who participated in the focus groups were aware of the relevance of good and secure mHealth systems for improving the treatment of patients. They failed to detect relevant barriers in using new technologies involving the use of smartphone applications, for the management of bipolar disorder. However, the participants were concerned about safety, privacy, and ethical issues related to sharing personal information. The users were also concerned about the possibility of future electronic devices acting as substitutes for actual face-to-face networking.

The five main "take home" recommendations that need to be considered when developing the mHealth device can be summed 
up by quoting a few brief comments made by some of the participants:

- "The involvement of informal caregivers is needed to provide trust and hope to patients."

- "Patients need to be adequately involved in both real and virtual social networks."

- "The appropriate flow and management of the information is the most relevant issue."

- "The perception of having direct access to clinicians makes patients feel completely safe."

- "It is important that the use of the applications does not substitute human care."

The issues raised by the participants of Trento and Sabadell were largely the same. However, while in Trento, the comments and recommendations that emerged during the focus groups were more oriented toward social networking-related issues, in Sabadell, the participants' comments were primarily focused on monitoring and alert systems. Although the findings of this study cannot be generalized, they may be transferred to similar situations.

The inputs obtained from the focus groups were used to formulate mHealth requirements for the preparation of the call for tender. These requirements explicitly indicate the relevant aspects that companies need to consider during the preparation of their proposals. The call for tender of the NYMPHA-MD project will be made available through the project website at www.nymphamd-project.eu, once tender has been revised and approved by the European Commission and all the legal departments of the procurers involved in the project.

\section{ACKNOWLEDGMENTS}

The authors would like to acknowledge the partial support of the European Commission through NYMPHA-MD project grant No. 610462.

\section{REFERENCES}

[1] Wittchen HU, Jacobi F, Rehm J, Gustavsson A, Svensson M, Jönsson B, Olesen J, Allgulander C, Alonso J, Faravelli C, Fratiglioni L, Jennum P, Lieb R, Maercker A, van Os J, Preisig M, Salvador-Carulla L, Simon R, Steinhausen HC. The size and burden of mental disorders and other disorders of the brain in Europe 2010. Eur Neuropsychopharmacol. 2011 Sep 2;21(9):655-79.

[2] Faurholt-Jepsen M, Vinberg M, Christensen EM, Frost M, Bardram J, Kessing LV. Daily electronic self-monitoring of subjective and objective symptoms in bipolar disorder-the MONARCA trial protocol (MONitoring, treAtment and pRediCtion of bipolAr disorder episodes): a randomised controlled single-blind trial. BMJ Open. 2013 Jul 24;3(7).

[3] Gabbard GO. Psychotherapy in psychiatry. International Review of Psychiatry. 2007;19(1): 5-12.
[4] Burns, Tom (2006). Psychiatry: A very short introduction. Oxford University Press. ISBN 9780192807274.

[5] Proudfoot JG, Jayawant A, Whitton AE, Parker G, Manicavasagar V, Smith M, Nicholas J. Mechanisms underpinning effective peer support: a qualitative analysis of interactions between expert peers and patients newlydiagnosed with bipolar disorder. BMC Psychiatry. 2012 Nov 9;12:196.

[6] Thornicroft G, Tansella M. Growing recognition of the importance of service user involvement in mental health service planning and evaluation. Epidemiol Psichiatr Soc. 2005 Jan-Mar;14(1):1-3.

[7] Tait L, Lester H. Encouraging user involvement in mental health services. Advances in Psychiatric Treatment. 2005;11:168-75.

[8] Donker T, Petrie K, Proudfoot J, Clarke J, Birch MR, Christensen H. Smartphones for smarter delivery of mental health programs: a systematic review. J Med Internet Res. 2013 Nov 15;15(11):e247. doi: 10.2196/jmir.2791.

[9] Faurholt-Jepsen M, Vinberg M, Frost M, Christensen EM, Bardram J, Kessing LV. Daily electronic monitoring of subjective and objective measures of illness activity in bipolar disorder using smartphones - the MONARCA II trial protocol: a randomized controlled single-blind parallel-group trial. BMC Psychiatry. 2014 Nov 25;14(1):309.

[10] Mayora O, Frost M, Arnrich B, Gravenhorst F, Grunerbl A, Muaremi A, Osmani V, Alessandro Puiatti A, Reichwaldt N, Scharnweber C, Troster G. Mobile Health Systems for Bipolar Disorder: The relevance of Non-Functional Requirements in MONARCA Project. IGI International Journal of Handheld Computing Research. 2014.

[11] Flores JG, Alonso CG. Using focus groups in educational research. Evaluation Review. 1995;19(1): 84-101.

[12] Gabrielli S, Mayora O, Bardram J, Marcu G. Co- Designing Personal HealthCare Solutions for the Treatment of Bipolar Disorder, Nordi CHI 2010 Workshop on Therapeutic Strategies - a Challenge for User Involvement in Design, on October 17th, 2010.

[13] Bardram JE, Frost M, Szántó K, Faurholt-Jepsen M, Vinberg M, Kessing LV. Designing mobile health technology for bipolar disorder: a field trial of the MONARCA system. In Proceedings of the SIGCHI Conference on Human Factors in Computing Systems. 2013, 2627-2636.

[14] Marcu G, Bardram J, Gabrielli S. A Framework for Overcoming Challenges in Designing Persuasive Monitoring Systems for Mental Illness. Pervasive Health Conference, May 2011, Ireland. 